\title{
REVISÃO RELACIONADA A ALGUNS ASPECTOS TÉCNICOS E FISIOLÓGICOS DAS ONDAS F E ANÁLISE DOS DADOS OBTIDOS EM UM GRUPO DE INDIVÍDUOS DIABÉTICOS
}

\author{
João Antonio Maciel Nobrega ${ }^{1}$, Gilberto Mastrocola Manzano ${ }^{2}$
}

\begin{abstract}
RESUMO - Fazemos uma revisão da literatura e discutimos aspectos fisiológicos relacionados aos tipos de neurônios motores envolvidos na gênese das ondas F. São revistos aspectos técnicos relativos ao número de ondas $\mathrm{F}$ a serem colhidas para uma melhor definição dos parâmetros utilizados no estudo destes potenciais. São analisadas as latências, persistência e as velocidades de condução das ondas $F$ registradas nos nervos ulnares em um grupo de indivíduos normais e em um grupo de pacientes diabéticos.
\end{abstract}

PALAVRAS-CHAVE: ondas F, condução nervosa.

\begin{abstract}
A review of technical and physiological aspects of F-wave studies and analyzis of the data obtained in a group of diabetic patients

ABSTRACT - We reviwed some physiological aspects of the F-wave studies, mainly related to the motoneurone sizes involved in the generation of this potentials and the number of stimuli necessary to analyze the F-wave parameters. F-wave latencies and F-wave conduction velocities obtained in a group of normal volunteers and in a group of diabetic patients are analyzed.
\end{abstract}

KEY WORDS: F-waves, neural conduction.

A onda $\mathrm{F}$ é definida como uma resposta motora tardia resultante da ativação antidrômica de um ou de um pequeno número de neurônios motores após a estimulação elétrica de um nervo periférico' ${ }^{1}$. Magladery e McDougal ${ }^{2}$ foram os primeiros autores a relatar no músculo abdutor do $5^{0}$ dedo da mão, após estimulação do nervo ulnar, a ocorrência de um potencial com latência maior que aquela da onda $\mathrm{M}$ e o designaram onda F. Notaram que este potencial aumentava de amplitude com o aumento da intensidade de estimulação até atingir a amplitude máxima com estimulação supramáxima. Também observaram que essas ondas variavam de amplitude de indivíduo para indivíduo e que, no mesmo indivíduo, variavam as amplitudes, as morfologias e as latências em estímulos subsequentes, e que nem todos os potenciais de ação musculares compostos (PAMC) eram seguidos de um potencial de onda $F$. Chamaram ainda a atenção para o fato de que pon- tos de estimulação mais distais levavam a redução da latência das ondas $\mathrm{M}$ e a um aumento das latências das ondas $\mathrm{F}$ em relação aos pontos de estímulação mais proximais. Esta redução da latência da onda M era evidente com a aproximação do ponto de estímulo ao ponto de registro; por outro lado, o aumento da latência da onda $F$ sugeria que o espaço a ser percorrido pelo impulso para atingir o músculo seria maior. Outro fato interessante é que estímulos aplicados ao nervo ulnar distalmente a um ponto de bloqueio desse nervo por procaína não elicitavam ondas F. Aventaram que a ocorrência desta onda poderia ser explicada por dois mecanismos: o primeiro, por uma ativação retrógrada das células do corno anterior e o segundo por um mecanismo reflexo envolvendo uma conexão central e via aferente e eferente distinta. Como a análise das medidas de condução motora por estímulos em dois pontos é diferente daquela registrada no mesmo

\footnotetext{
Setor de Neurofisiologia Clínica da Disciplina de Neurologia da Universidade Federal de São Paulo (UNIFESP). 'Professor associado; ${ }^{2}$ Professor substituto.
}

Recebido 18 Novembro 2000. Aceito 20 Dezembro 2000.

Dr. João Antonio Maciel Nobrega - Rua Pedroso Alvarenga 1062 / 54 - 04531-004 São Paulo SP - Brasil. FAX $55113167-3371$. E-mail: jamnobrega@aol.com 
segmento através da onda $F$, sugeriram que esta onda seria componente de um reflexo. Após a descrição original de Magladery e McDougal ${ }^{2}$ vários autores passaram a estudar a ocorrência das ondas $\mathrm{Fe} \mathrm{a}$ sugerir os mecanismos relacionados a sua gênese. Alguns sugeriam que a onda $F$ fosse a expressão de um reflexo polissináptico como Hagbarth ${ }^{3}$, que se baseou em experimentos em que aumentava a excitabilidade dos motoneurônios por uma contração muscular ou tetanização do músculo no qual registrava a onda F. Liberson ${ }^{4}$, também defendeu esta idéia baseado no estudo das velocidades de condução das vias aferentes e eferentes. Uma segunda explicação foi defendida por outra corrente que entendia a onda $\mathrm{F}$ como uma resposta de um motoneurônio ativado por um mecanismo antidrômico, ou seja, a ativação retrógrada do motoneurônio pelo impulso antidrômico que aporta ao corpo celular após uma estimulação elétrica e que, eventualmente, geraria disparo, o qual se propagaria em direção ortodrômica. Gassel e Wiesendanger ${ }^{5}$, baseados em estudos de deaferentação de membros de gatos, concluíram que as ondas $\mathrm{F}$ eram geradas por descargas induzidas por estímulos antidrômicos. A conclusões semelhantes chegaram Mcleod e Wray ${ }^{6}$ em um estudo com macacos submetidos a deaferentação, e Miglietta ${ }^{7}$, estudando quatro pacientes paraplégicos submetidos a mielotomia para redução de espasticidade, que mostraram que as ondas $F$ persistiam após deaferentação. Mayer e Feldman ${ }^{8}$ chegaram a conclusão semelhante também através do estudo de pacientes deaferentados. Thorne ${ }^{9}$, analisando respostas tardias em músculos da mão, pela comparação das ondas $M$ relacionadas às ondas $F$, concluiu que estas decorrem da ativação antidrômica de uma pequena percentagem de neurônios motores. Através da técnica da eletromiografia de fibra única Trontelj ${ }^{10}$ demonstrou que a latência e o "jitter" de uma determinada onda $F$ praticamente não variam, sugerindo que esta onda não é componente de um reflexo. Este conjunto de fatos sugere que o estímulo responsável pela geração da onda $\mathrm{F}$ caminha antidrômicamente até a célula do corno anterior da medula ativando algumas células e evocando potenciais, os quais percorrem ortodrômicamente as fibras motoras, levando à geração das ondas F. Renshaw ${ }^{11}$ mostra que, após secção da raiz posterior em gatos, a estimulação de um nervo motor gera um impulso antidrômico no sentido do sistema nervoso central. A seguir, há geração de potencial com um retardo central de aproximadamente $1 \mathrm{~ms}$ e que corresponde ao potencial que irá produzir a onda $\mathrm{F}$.
Para que se possam compreender os mecanismos envolvidos na geração das ondas F é de fundamental importância uma breve revisão da anatomia e da fisiologia da unidade motora. Uma unidade motora é constituída de uma célula, o motoneurônio, cujo corpo celular situa-se no corno anterior da medula ou em núcleos motores no tronco cerebral. Esta célula apresenta varias projeções que são os dendritos e uma projeção maior, o axônio, que se dirige para fora do sistema nervoso central e que, após ramificações distais, estabelece sinapses com fibras musculares inervadas por este neurônio. A este conjunto neurônio motor, axônio e as fibras musculares por ele inervadas dá-se o nome de unidade motora. Uma pequena porção do segmento inicial do axônio (SIA) não é mielinizada e neste ponto o limiar de disparo é a metade do que o observado nas porções mielinizadas ${ }^{12}$. O corpo celular e os dendritos dos motoneurônios contêm inúmeras sinapses com funções excitatórias ou inibitórias. A resultante destes impulsos excitatórios e inibitórios determina a excitabilidade do neurônio. Logo após o SIA, alguns motoneurônios possuem um ramo colateral que faz sinapse com um interneurônio inibidor, a célula de Renshaw. A ativação da célula de Renshaw por um impulso antidrômico, trafegando centripetamente no sentido do corpo do neurônio motor, gera um impulso inibitório que poderá bloquear o respectivo neurônio motor, impedindo a geração de onda $\mathrm{F}^{13}$. As condições de excitação do motoneurônio dependem da resultante das influências excitatórias e inibitórias geradas pelo sistema nervoso central e periférico. Quando um nervo periférico misto é submetido a um estímulo supramáximo o potencial de ação motor antidrômico gerado entra pela raiz ventral encontrando o corpo celular dos respectivos neurônios motores em vários níveis de excitabilidade, níveis estes de que dependerá a geração ou não de despolarização recorrente de cada neurônio motor, conseqüentemente gerando ou não ondas F. Suponha-se que o potencial de ação antidrômico atravesse o SIA. Neste caso ocorrerá despolarização do corpo celular que se propagará pelos dendritos que compõem o neurônio. É possível também supor que, em um dado momento, dependendo das condições iniciais de excitabilidade do motoneurônio, enquanto os dendritos ainda estão despolarizados, o SIA já se repolarizou. Não se encontrando mais no período refratário, a diferença de potencial entre as porções despolarizadas dos dendritos e o SIA geram correntes capazes de despolarizar suficientemente este ultimo levando 
então à propagação de um novo impulso elétrico ortodromico, que caminha para as fibras musculares componentes desta unidade motora, despolarizando-as e gerando a onda F. Em alguns momentos, a despolarização do corpo celular e dendritos pode se fazer mais rapidamente, gerando um circuito local ainda com o SIA em período refratário e assim não será gerada a onda F. Outro mecanismo de bloqueio da onda $\mathrm{F}$ decorre do fato de que a transição entre a porção mielinizada do axônio e o SIA não favorece a condução para o corpo celular do neurônio, porque aqui a corrente seria dificultada pela condução contínua e se em alguns momentos o potencial da membrana do SIA estiver hiperpolarizado por influências segmentares ou suprasegmentares, o potencial gerado distalmente não cruzará este segmento e, portanto, não produzira as condições necessárias para geração de onda F. Estes fatores variam de momento a momento, motivo pelo qual a cada estímulo obtêm-se ondas $F$ geradas por subgrupos diferentes de motoneurônios. Um fato a ser considerado é que a amplitude da onda $F$ sendo significativamente menor que o da onda $M$ sugere que aquela onda é produzida por um menor número de neurônios motores ativados do que para a geração da onda M. Em indivíduos normais, a amplitude da onda F corresponde a 1-5\% da amplitude da onda $\mathrm{M}^{14}$. A análise individual das ondas $\mathrm{F}$ sugere que cada uma delas é gerada por 1 a 3 unidades motoras ${ }^{9,15}$.

Fator polêmico são quais os tipos de neurônios motores responsáveis pela geração de ondas $\mathrm{F}$. Alguns autores ${ }^{15,16}$ sugerem que essas ondas seriam geradas preferencialmente por neurônios motores grandes, hipótese não compartilhada por outros ${ }^{17-19}$ que entenderam não haver especificidade dos neurônios geradores de ondas F. Dentre estes, Kimura e col. ${ }^{17}$, apresentam um estudo com três series de experimentos nos quais estimulam seletivamente grupos de neurônios motores através da técnica de estimulação sublimiar ou da técnica de colisão e concluem que tanto neurônios motores maiores quanto menores podem gerar ondas F. Conclusão semelhante é feita por Dengler e col. ${ }^{18}$, que analisam os neurônios geradores de ondas $F$ registrando além dos potenciais das ondas $\mathrm{M}$ e $\mathrm{F}$ a força de contração das unidades motoras envolvidas. Algumas possibilidades são citadas para explicar por que os neurônios motores maiores seriam os responsáveis pelas geração das ondas $\mathrm{F}$. Uma delas é de que os neurônios motores maiores, através dos colaterais para as células de Renshaw, inibem motoneurônios menores ${ }^{15,20}$. Este fato ocorre porque o impulso antidrômico percorre mais rapidamente as fibras de maior diâmetro dos neurônios motores maiores atingindo mais rapidamente as células de Renshaw que os impulsos dos neurônios motores menores, bloqueando, portanto, estas células. Outra hipótese é que é mais fácil para as células de Renshaw inibirem os neurônios motores menores, uma vez que há uma menor superfície de membrana celular a ser bloque$\mathrm{ada}^{21}$. Outro mecanismo seria que as fibras aferentes, que fazem sinapses com os neurônios motores menores, conduzem mais rapidamente que as fibras motoras, de maneira tal que o impulso conduzido pelas fibras aferentes, atingem primeiramente o corpo celular do neurônio motor, gerando despolarização e um potencial de ação que será bloqueado por colisão com a despolarização antidrômica conduzida pelas fibras motoras mais lentas e que ainda não atingiram o soma ${ }^{15}$. Outra hipótese a ser considerada é que neurônios motores menores possuem potencial de repouso mais próximo do limiar de disparo. Isto os leva a despolarizarem rapidamente a soma e os dendritos antes que os SIA deixem de estar em período refratário e, consequentemente, os impulsos são bloqueados neste ponto. Komori e col. ${ }^{22}$ referem haver uma grande correlação entre o tamanho das unidades motoras e a latência das ondas F. Quanto maior a unidade motora menor a latência e, portanto, maior a velocidade de condução.

Para o estudo das ondas F utiliza-se estimulação supramáxima, para que todos os axônios sejam despolarizados e para evitar a contaminação da reposta tardia pelo reflexo $\mathrm{H}$ assim como ativação voluntária de unidades motoras ${ }^{23}$.

Os principais parâmetros analisados no estudo das ondas F são:

1 - Morfologia: a morfologia das ondas F obtidas em um mesmo nervo varia de estímulo a estímulo, pois elas são geradas por grupos diferentes de neurônios motores, à exceção das ondas "repetiti7vas", que possuem a mesma morfologia e latência, uma vez que se considera como produzidas por um único neurônio motor;

2 - Amplitude: é variável com o subgrupo de unidades motoras ativadas para gerar cada onda F. Em geral de 1 a $5 \%{ }^{14}$ da amplitude da onda M. Alguns autores definem um limite mínimo de amplitude para considerar-se um potencial onda $F$, no sentido de evitar que o ruído seja considerado potencial. Este limite é variável. Kiers e col. ${ }^{24}$ e Fisher ${ }^{25}$ consideram $20 \mathrm{uV}$ enquanto outros autores $40 \mathrm{uV} \mathrm{V}^{26,27}$. Há também autores que não consideram necessário definir um limite ${ }^{1}$. 
3 - Duração: variável com o subgrupo de neurônios motores ativados;

4 - Latência: é o parâmetro mais freqüentemente analisado nos estudos de onda $\mathrm{F}$ na rotina clínica. Deve-se considerar que como existem motoneurônios com condução mais rápida e outros com condução mais lenta haverá uma latência mínima que representa a condução nas fibras mais rápidas e uma latência máxima, que representa a condução nas fibras mais lentas. Com estes dados pode-se determinar a latência média e a dispersão, que representa a diferença entre a latência mínima e a latência máxima. As latências têm intima relação com a altura do indivíduo e o tamanho do segmento estudado. Para superar este fato pode-se transformar as latências em velocidades através da fórmula distância/ [(Latência-1)/2]. Desta maneira obter-se-ão a velocidade mínima, a máxima e a média. PeioglouHarmoussi e col. ${ }^{27}$, não encontraram diferenças significantes dos valores das latências entre lados no mesmo indivíduo;

5 - Cronodispersão: a diferença de latência entre as ondas $\mathrm{F}$ indica que os estímulos trafegam nos axônios com diferentes velocidades e estas são diretamente proporcionais ao diâmetro das fibras, e, portanto, ao tamanho da unidade motora ${ }^{16}$. A diferença de latência entre as respostas evocadas pelas fibras mais rápidas e pelas mais lentas é chamada de cronodispersão que, segundo alguns autores, é de grande importância no diagnóstico precoce de neuropatias periféricas ${ }^{16,28}$;

6 - Persistência: é definida como o número de vezes que se obtêm ondas $F$ após um determinado número de estímulos e é usualmente expressa em percentual. Alguns autores referem ser este um parâmetro de grande importância para o diagnóstico de bloqueio proximal de condução ${ }^{15,25}$. Em indivíduos normais varia de nervo para nervo. Por exemplo, em membros superiores varia de $60 \%$ a $100 \%$ no nervo mediano e de $70 \%$ a $100 \%$ no nervo ulnar ${ }^{15,25}$. Fator importante para a interpretação da persistência é a fixação ou não de limite de amplitude para a determinação das ondas F. O estudo da persistência da onda $F$ pode ser o primeiro ou o único parâmetro a alterar-se em algumas doenças como a síndrome de Guillain-Barré1.

A manobra de Jendrassic também pode ser utilizada para facilitar o aparecimento de ondas $F^{19,29}$. Fraser e Olney ${ }^{29}$ mostram em seu trabalho que manobras de facilitação aumentam a persistência e reduzem a latência mínima das ondas F. Por outro lado há autores que fazem restrições a estas manobras ${ }^{23.30}$.
Outro ponto que gera discussão é o número necessário de ondas $\mathrm{F}$ a serem analisadas para que os parâmetros obtidos sejam os mais precisos possíveis. Como as latências variam em estímulos sucessivos e a persistência também varia de indivíduo para indivíduo em um mesmo nervo, é fundamental coIher-se um número de ondas $F$ suficiente para que os parâmetros registrados sejam o mais representativo possível da população de ondas que o nervo em estudo pode gerar. Em outras palavras, seria o número de ondas $\mathrm{F}$ suficiente para que se possam registrar as unidades mais rápidas e as mais lentas que compõem o nervo em análise e que colaboram para a geração de ondas F. Observa-se que conforme se aumenta o número de ondas analisadas, o valor da latência mínima se reduz, da latência máxima aumenta e como conseqüência aumenta a cronodiperssão. Alguns autores analisam em seus trabalhos um determinado número de estímulos ${ }^{24,29}$ ao passo que outros fixam um número de ondas a serem analisadas aplicando tantos estímulos quantos necessários ${ }^{25,27}$. Há discussão quanto ao número ideal de ondas a serem analisadas. Há trabalhos que citam 5 ondas como suficiente ${ }^{31}, 10$ ondas $^{32}$, ou um número maior ${ }^{16,33}$. Chroni e col. ${ }^{34}$ analisam a importância do tamanho da amostra para determinar os parâmetros das latências das ondas $F$ no nervo ulnar e citam como número adequado a coleta de 40 ondas. Alguns autores chamam a atenção que este número pode ser diferente para indivíduos normais e para pacientes com neuropatias ${ }^{1,34}$. Uma possível explicação é que devido à perda de unidades motoras e redução da persistência, é necessária a análise de um maior número de ondas para obter-se dados mais representativos da totalidade de motoneurônios geradores de ondas F. Chroni e col..$^{35}$ analisando ondas $\mathrm{F}$ no nervo peroneo concluem que para a obtenção de uma medida acurada o menor número de ondas necessário é para a latência média e o maior número para a cronodispersão. Também concluem que em alguns parâmetros é necessária a coleta de um maior número de ondas em pacientes portadores de polineuropatia do que em indivíduos sadios e sugerem que 40 ondas seriam suficientes para obter-se um valor acurado para todos os parâmetros analisados. Fisher ${ }^{25}$ sugere que 10 a 20 ondas F seriam um número razoável para uso clínico. Nobrega et al. ${ }^{36}$ mostram que para a latência média 5 estímulos já são suficientes, porém para a determinação das latências máxima e mínima são necessários um mínimo de 16 estímulos e para a cronodispersão um mínimo de 20 estímulos, tanto para a análise 
Tabela 1. Média e desvio padrão dos parâmetros obtidos nos nervos ulnares dos 23 indivíduos normais analisados.

\begin{tabular}{|c|c|c|c|c|c|c|}
\hline $\begin{array}{l}\text { Latência } \\
\text { mínima (ms) }\end{array}$ & $\begin{array}{c}\text { Latência } \\
\text { máxima (ms) }\end{array}$ & $\begin{array}{c}\text { Latência } \\
\text { média (ms) }\end{array}$ & $\begin{array}{l}\text { Cronodispersão } \\
\text { (ms) }\end{array}$ & Persistência & $\begin{array}{c}\text { Velocidade de condução } \\
\text { máxima }(\mathrm{m} / \mathrm{s})\end{array}$ & $\begin{array}{c}\text { Velocidade de condução } \\
\text { mínima }(\mathrm{m} / \mathrm{s})\end{array}$ \\
\hline $25,5 \pm 2,2$ & $29,6 \pm 2,2$ & $27,0 \pm 2,2$ & $4,1 \pm 1,3$ & $95 \%$ & $65,1 \pm 3,8$ & $54,5 \pm 3,4$ \\
\hline
\end{tabular}

Tabela 2. Média e desvio padrão dos parâmetros obtidos nos nervos ulnares dos 27 pacientes diabéticos analisados.

\begin{tabular}{lcccccc}
\hline $\begin{array}{l}\text { Latência } \\
\text { mínima }(\mathrm{ms})\end{array}$ & $\begin{array}{c}\text { Latência } \\
\text { máxima }(\mathrm{ms})\end{array}$ & $\begin{array}{c}\text { Latência } \\
\text { média }(\mathrm{ms})\end{array}$ & $\begin{array}{c}\text { Cronodispersão } \\
(\mathrm{ms})\end{array}$ & Persistência & $\begin{array}{c}\text { Velocidade de condução } \\
\text { máxima }(\mathrm{m} / \mathrm{s})\end{array}$ & $\begin{array}{c}\text { Velocidade de condução } \\
\text { mínima }(\mathrm{m} / \mathrm{s})\end{array}$ \\
\hline $28,9 \pm 2,6$ & $34,6,6 \pm 3,9$ & $30,9 \pm 2,7$ & $5,7 \pm 2,5$ & $81 \%$ & $55,2 \pm 3,9$ & $44,8 \pm 4,7$ \\
\hline
\end{tabular}

dos indivíduos normais como dos diabéticos. Estes autores ressaltam que estes números são suficientes para a avaliação de grupos mas não para a análise individual.

\section{MÉTODO}

Foi estudado um grupo de voluntários normais e um de pacientes diabéticos. 0 primeiro grupo constou de 23 indivíduos, 7 do sexo masculino, cuja faixa etária variou de 17 a 59 anos. Estes indivíduos não apresentavam patologias que pudessem causar neuropatia periférica e não estavam em uso de medicamentos potencialmente neurotóxicos. 0 segundo grupo foi formado por 27 pacientes, 8 do sexo masculino, com diabetes do tipo I, todos com acompanhamento clínico e metabólico pela Disciplina de Endocrinologia da Universidade Federal de São Paulo (UNIFESP-EPM), com faixa etária variando de 17 a 55 anos. Os indivíduos que compõem o grupo de normais foram selecionados e encaminhados pela Disciplina de Endocrinologia bem como os pacientes diabéticos (um normal e um diabético cada dia), tentando-se manter idades e alturas semelhantes nos dois grupos. Foram determinadas as latências mínima, máxima e média assim como a persistência e as velocidades de condução máxima e mínima das ondas $\mathrm{F}$ registradas nos nervos ulnares direito dos indivíduos destes grupos. Para o registro das ondas $\mathrm{F}$ no nervo ulnar foram aplicados 64 estímulos e a metodologia empregada está descrita na literatura ${ }^{36}$.

\section{RESULTADOS}

Os resultados obtidos para as latências máxima, média e mínima das ondas $\mathrm{F}$ registradas nos nervos ulnares assim como da persistência e das velocidades de condução máxima e mínima das ondas $F$ registradas no grupo de normais estão na Tabela $1 \mathrm{e}$ no grupo de diabéticos na Tabela 2 .

\section{DISCUSSÃO}

A análise dos dados obtidos comparando grupos mostra alterações significativas para todos os parâmetros. As latências (mínima, média e máxima) assim como a crondisperssão mostram-se significativamente aumentadas em relação ao grupo controle, evidenciando um comprometimento do sistema nervoso periférico no grupo de diabéticos. Estes dados confirmam que o estudo das ondas F é uma ferramenta de valor no diagnóstico da neuropatia diabética.

\section{REFERÊNCIAS}

1. Panayiotopoulos $\mathrm{CP}$, Chroni E. F-waves in clinical neurophysiology: a review, methodological issues and overall value in peripheral neuropathies. Electroencephalogr Clin Neurophysiol 1996;101:365-374.

2. Magladery JW, McDougal DB. Electrophysiological studies of nerve and reflex activity in normal man. Identification of certain reflexes in the electromyogram and the conduction velocity of peripheral nerve fibers. Bull. Johns Hopkins Hosp 1950;86:265-290.

3. Hagbarth KE. Post-tetanic potentiation in myotatic reflexes in man. J Neurol Neurosurg Psychiatry 1962;25:1-10.

4. Liberson WT. Sensory conduction velocities in normal individuals and in patients with peripheral neuropathies. Arch Phys Med Rehabil 1963;44:313-320.

5. Gassel MM, Wiesendanger M. Recurrent and reflex discharges in plantar muscles of the cat. Acta Physiol Scand 1965;65:138-142.

6. Mcleod JG, Wray S. An experimental study of the $f$ wave in baboon. J Neurol Neurosurg Psychiatry 1966;29:196-200.

7. Miglietta OE. The F reponse of transverse myelotomy. In Desmedt JE (ed). New developments in electromyography and clinical neurophysiology. Basel: Karger, 1973:323-327.

8. Mayer RF, Feldman RG. Observations on the nature of $f$ wave in man. Neurology 1967;17:147-156.

9. Thorne J. Central responses to electrical activation of the peripheral nerves supplying the intrinscic hand muscles. J Neurol Neurosurg Psychiatry 1965;28:482-495.

10. Trontelj JV. A study of the F-response by single fibre electromyography. In Desmedt, JE. New developments in electromyograghy and clinical neurophysiology, Basel: Karger, 1973:318-323.

11. Renshaw B. Central effects of centripetal impulses in axons of spinal ventral roots. J.Neurophysiol 1946;9:191-204.

12. Dumitru D. Special nerve conduction techniques. In: Electrodiagnostic medicine. Philadelphia: Hanley \& Belfus 1995:177-209.

13. Eccles JC, Fatt P, Koketsu K. Cholinergic and inhibitory synapses in a pathway from motor axon collaterals to motor neurones J Physiol 1954;126:524-562.

14. Kimura J. In: Electrodiagnosis in diseases of nerve and muscle: principles and practice. Philadelphia: Davis, 1989:332-355

15. Guillof RJ, Moderres-Sadeghi H. Preferencial generation of recurrent responses by groups of motorneurons in man. Brain 1991;114:1771-1801.

16. Panayiotopoulos CP. F Chronodispersion: a new electrophysiologic method. Muscle Nerve 1979;2:68-72. 
17. Kimura J, Yanagisawa H, Yamada T, Akihisa M, Sasaki H, Kimura A. Is the $\mathrm{F}$ wave elicited in a select group of motor neurons?. Muscle Nerve 1984;7:392-399.

18. Dengler R, Kossev A, Wohlfahrt K, Schubert M, Elek J, Wolf W. F waves and motor unit size. Muscle Nerve 1992;15:1138-1142.

19. Doherty TJ, Komori T, Stashuk DW, Kassam A, Brown WF. Physiological properties of single thenar motor units in the F-response of younger and older adults. Muscle Nerve 1994;17:860-872.

20. Eccles JC. The central action of antidrômical impulses in motor nerve fibers Pflugers Arch 1955;260:385-415.

21. Eccles JC, Eccles RM, Iggo A, Ito M. Distribution of recurrent inhibition among motoneurones. J Physiol 1961;159:479-499.

22. Komori T, Watson BV, Brown WF. Characteristics of single " $F$ " motor units at diferent stimulus intensities. Muscle Nerve 1991;14:875.

23. Kimura JF. Wave velocity in the central segment of the median and ulnar nerves: a study in normal subjects and in patients with charcotmarie-tooth disease. Neurology 1974;24:359-546.

24. Kiers L, Clouston P, Zuniga G, Cros D. Quantitative studies of $f$ responses in guillain-barré syndrome and chronic inflammatory demyelinating polyneuropathy. Electroencephalogr Clin Neurophysiol 1994;93:255-264.

25. Fisher MA. H-reflexes and F-waves: physiology and clinical indications. Muscle Nerve 1992;15:1223-1233.

26. Macleod WN. Repeater F waves: a comparison of sensitivity with sensory antidromic wrist-to-palm latency and distal motor latency in the diagnosis of carpal tunnel syndrome. Neurology 1987;37:773-778.

27. Peioglou-Harmoussi S, Fawcett PRW, Howel D, Barwick DD. F response: a study of frequency, shape and amplitude characteristics in healty control subjects. J Neurol Neurosurg Psychiatry 1985;48:1159-1164

28. Chroni E, Panayiotopoulos CP. F tacheodispersion: quantitative analysis of motor fiber conduction velocities in patients with polyneuropathy. Muscle Nerve 1993;16:1302-1309.

29. Fraser JL, Olney RK. The relative diagnostic sensitivity of different $f$ wave parameters in various polyneuropathies. Muscle Nerve 1992;15:912-918.

30. Panayiotopoulos CP, Scarpalezos S, Nastas PE. F-wave studies on the deep peroneal nerve. J Neurol Sci 1977;31:319-329.

31. Marra TRF. Wave measurements: a comparison of various recording techniques in health and peripheral nerve disease. Electromyogr Clin Neurophysiol1987;27:33-37.

32. Delisa JA, Lee HJ, Baran EM, Siu Lai K, Spielholz N, Mackenzie K. Manual of nerve conduction velocity and clinical neurophysiology. 3.Ed. New York: Raven Press, 1994.

33. Peioglou-Harmoussi S, Howel D, Fawcett PRW, Barwick DD. F response behaviour in a control population. J Neurol Neurosurg Psychiatry 1985;48:1152-1158.

34. Chroni E, Taub N, Panayiotopoulos CP. The importance of sample size for the estimation of $\mathrm{f}$ wave latency parameters in the ulnar nerve. Muscle Nerve 1994;17:1480-1483.

35. Chroni E, Taub N, Panayiotopoulos CP. The importance of sample size for the estimation of $\mathrm{f}$ wave latency parameters in the peroneal nerve. Electroencephalogr Clin Neurophysiol 1996;101:375-378.

36. Nobrega JAM, Manzano GM, Novo NF, Monteagudo PT. Sample size and the study of F-waves. Muscle Nerve 1999; 22: 1275-1278. 